Research Paper

\title{
No Significant Detectable Anti-infection Effects of Aspirin and Statins in Chronic Obstructive Pulmonary Disease
}

\begin{abstract}
Josef Yayan ${ }^{凶}$
Department of Internal Medicine, Division of Pulmonary, Allergy and Sleep Medicine, Saarland University Medical Center, Homburg/Saar, Germany.

$\triangle$ Corresponding author: Dr. Josef Yayan, Department of Internal Medicine, Division of Pulmonary, Allergy and Sleep Medicine, Saarland University Medical Center, Kirrberger Straße. D-66421 Homburg/Saar, Germany. Tel +49 68411621620 Fax +49 68411623602 E-mail josef.yayan@hotmail.com.

๑ 2015 Ivyspring International Publisher. Reproduction is permitted for personal, noncommercial use, provided that the article is in whole, unmodified, and properly cited. See http://ivyspring.com/terms for terms and conditions
\end{abstract}

Received: 2014.11.13; Accepted: 2015.01.30; Published: 2015.03 .02

\begin{abstract}
Background: Past studies have shown that aspirin and statins decrease the rate and severity of exacerbation, the rate of hospitalization, and mortality in chronic obstructive pulmonary disease (COPD). Although these studies are relatively new, there is evidence that new therapeutic strategies could prevent exacerbation of COPD.

Trial design: This article examines retrospectively the possibility of using aspirin and statins to prevent exacerbation and infection in patients with COPD.

Methods: All patients with COPD were identified from hospital charts in the Department of Internal Medicine, Saarland University Medical Center, Germany, between 2004 and 2014.

Results: The study examined 514 medical reports and secured a study population of 300 with COPD. The mean age was $69 \pm 10$ years $(206$ men, $68.7 \%, 95 \% \mathrm{Cl}, 63.4-73.9 ; 94$ women, $31.3 \%$, $95 \% \mathrm{Cl}, 26.1-36.6)$. The study results did not show a causal relationship between aspirin and statins and prevention of exacerbation and infection in patients with COPD.

Conclusion: In contrast, in this study, the exacerbation and infection rates increased under medication with aspirin and statins $(\mathrm{p}=0.008)$.
\end{abstract}

Key words: aspirin, statins, infection, exacerbation, chronic obstructive pulmonary disease, pneumonia.

\section{Introduction}

Chronic obstructive pulmonary disease (COPD) is a preventable disease with additional pulmonary effects that may significantly influence its severity. The pulmonary component is characterized by airflow obstruction that is not fully reversible. The airflow limitation is usually progressive and is associated with a pathological inflammatory response of the lungs to noxious particles or gases. ${ }^{1,2}$ Pathological characteristics of COPD are inflammation of the small airways, called bronchiolitis, and damage to lung parenchyma resulting in emphysema. ${ }^{2}$ Clinical symptoms of COPD are coughing, sputum, and dyspnea. COPD can be categorized as mild, moderate, severe, and very severe depending on airflow limitation as measured with a spirometer. ${ }^{3}$ COPD is a chronic disease whose clinical progression may be characterized by exacerbation caused by unexpected factors worsening beyond probable daily deviations. ${ }^{4}$ Exacerbations have medical and predictive relevance, and they may result in marked functional and practical worsening. Preventing exacerbations and treating them appropriately are the main means of reducing morbidity. 4

Statins are hypolipemic medications with a recognized usefulness for avoiding cardiovascular diseases. Statins have anti-inflammatory effects in addi- 
tion to cholesterol-lowering properties. Observational studies have revealed that statins may be beneficial in reducing mortality from COPD. In addition, experimental studies on animals have demonstrated that statins have anti-inflammatory effects on lung tissue. ${ }^{5}$ Statins have been related to decreased hospitalizations due to COPD, indicating the possible advantageous effects of statins in patients with COPD. ${ }^{6}$ This new proposal suggests that statins have favorable effects on patients with COPD, which is a continuing inflammatory process. ${ }^{6}$

Treatment with aspirin has been linked to lower mortality rates in patients with COPD, 7 and aspirin has been reported to play a protective role in patients with COPD. ${ }^{7}$ A good outcome was found in patients with systemic inflammation who were treated with anti-platelet medication. ${ }^{8}$ These patients with COPD and pneumonia had shorter hospital stays and reduced need for intensive care. ${ }^{8}$

Recent insights into the relationship between COPD and the use of aspirin and statins raise new questions. The present study was conducted to determine the possible benefits of aspirin and statins in preventing inflammation and reducing exacerbation in patients with COPD. The study reviewed database

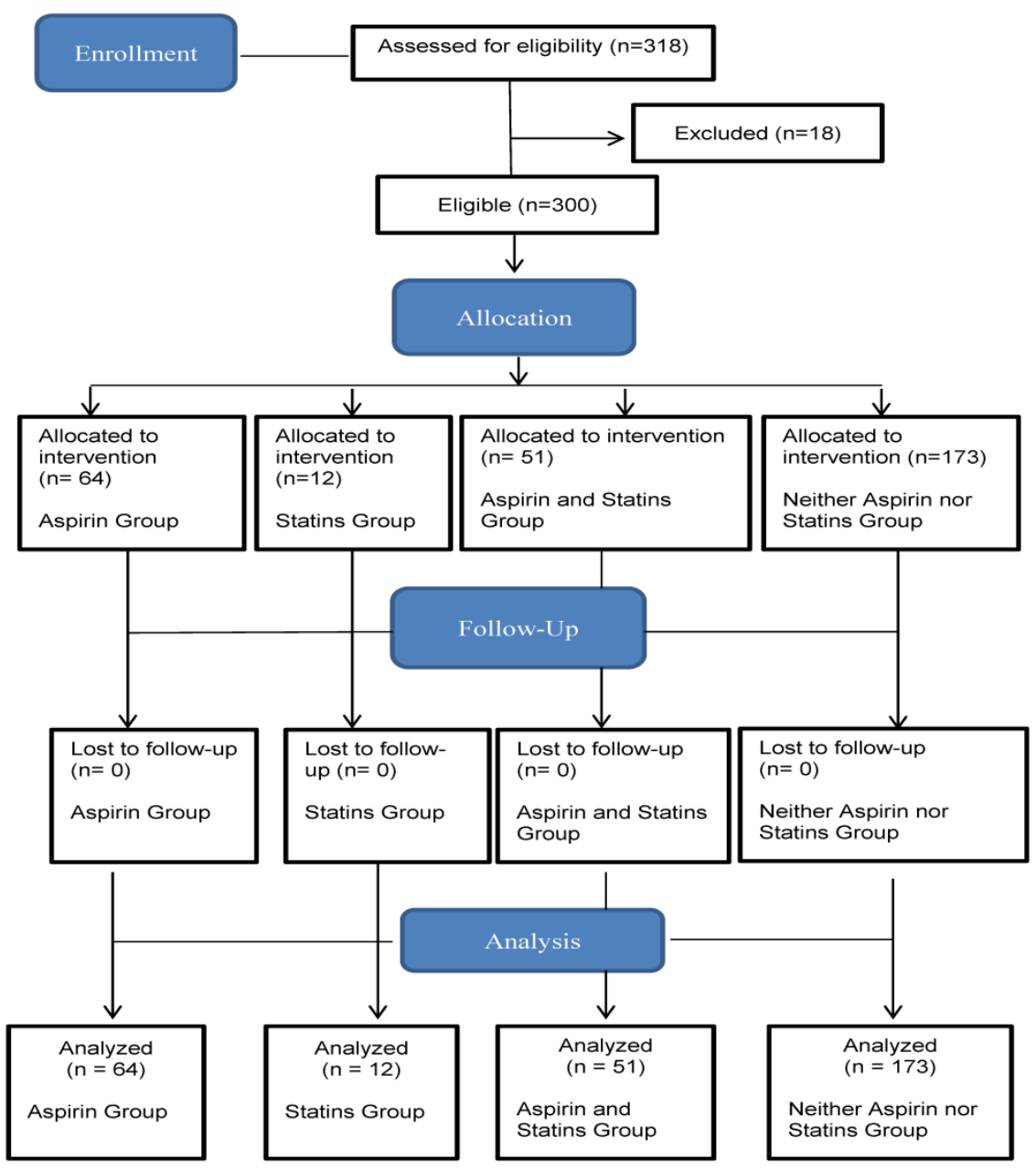

records of patients with COPD in the Department of Internal Medicine, Saarland University Medical Center, Germany. COPD was classified using the International Classification of Diseases (ICD). Patients were treated from 2004 to 2014 . This study sought to clarify whether patients with COPD treated with aspirin and statins had fewer infections and exacerbations compared to patients who took neither aspirin nor statins. In addition, the study examined the influence of COPD severity on the prevention of inflammation and exacerbation by using aspirin or statin.

\section{Material and methods}

\section{Patients}

This observational study examined retrospectively the amount of inflammation and number of exacerbations in patients with COPD with and without aspirin and statin use. The study used hospital chart data from the Department of Internal Medicine of the Saarland University Medical Center from 2004 to 2014. Patients with COPD were selected according to their family name by alphabetical ranking. The first 318 patients with COPD were included by alphabetical ranking according to their surnames in this study (Figure 1). Eighteen cases of patients' medical data were excluded because some patients occupied more than one group at the same time point during the study depending on newly identified indications or the discontinuation of aspirin and statins over the 10 -year study period. The study followed 300 patients with COPD first treated in 2004, noting the number of various acute infections and COPD exacerbations between January 1, 2004, and June 23, 2014.

Figure 1: Flow diagram 
Patients with COPD were categorized into four study groups: using aspirin, using statins, using aspirin and statins, and using neither aspirin nor statins. Care was taken that each study patient was grouped in only one study group for the entire study period. Indications for treatment with aspirin or statins were coronary artery disease, prior stroke, peripheral arterial occlusive disease, atrial fibrillation, deep vein thrombosis, hypercholesterolemia, and hyperlipidemia. This study's hypothesis requires that all periods of use and non-use reduce the frequency of infection. Accordingly, the researcher tracked at least one patient in each of the four study groups for the duration of the study period, depending on indications for administration of aspirin and statins or after discontinuation of aspirin or statins for various reasons. In all four study groups, the population was mixed in terms of age.

The infections investigated included acute exacerbations of COPD (ICD J44.0-J44.19), respiratory infections (ICD J20-J22), pneumonia (ICD J13-J10), acute urinary tract infection (ICD N39.0), erysipelas (ICD A46), sepsis (ICD A40.0-41.9), and other unspecified infections (ICD B99).

COPD symptoms were classified as an ongoing cough or a cough that produces a significant amount of mucus; shortness of breath, especially with physical activity; wheezing; and chest tightness. The diagnosis of manifested COPD was made with medical history, clinical examination, and lung function, as tested by bronchospasmolysis. COPD was diagnosed in the study population after discharge from the hospital. In each case, COPD was classified according to the latest edition of the ICD (ICD J44.0-J44.9).

To confirm the COPD diagnosis, all patients underwent spirometry. The mechanical properties of the lungs and lung volume were measured using body plethysmography $\left(\right.$ JAEGER $^{\circledR}$, MasterScreen ${ }^{\mathrm{TM}}$ Body, Germany). The inclusion criteria for the study were that patients with COPD had a history of inflammatory disease and exacerbations and had been examined with spirometry. The study excluded patients with COPD who had not been subjected to a lung test. The following were the inclusionary parameters of body plethysmography: forced expiratory volume in the first second (FEV1), vital capacity (VC), and Tiffeneau index (FEV1\%VC). A COPD diagnosis was based on lung function parameters only, according to the guidelines in the 2010 version of The Global Initiative for Chronic Obstructive Lung Disease (GOLD)..$^{9}$ COPD was identified mainly by a decrease in forced expiratory volume in one second and forced vital capacity ratio $<70 \%$ post-bronchodilators. According to the GOLD expert panel, COPD is classified into five stages, ranging from 0 to 4 . According to
GOLD, only stages 1 to 4 were considered for this study, because stage 0 (at risk) would comprise individuals with productive coughing and normal lung function. Mild COPD (GOLD 1) is defined by FEV1 $\geq$ $80 \%$, moderate COPD (GOLD 2) by FEV1 of between $50 \%$ and $80 \%$, severe COPD (GOLD 3) by FEV1 of between $30 \%$ and $50 \%$ predicted with or without chronic symptoms of cough and sputum production in stages 1 through 3, and very severe COPD (GOLD 4) by FEV $1 \leq 30 \%$ predicted and chronic respiratory failure. Chronic respiratory failure was classified as long-term hypoxemia caused by low blood oxygen levels or long-term hypercapnic respiratory failure due to high carbon dioxide blood levels.

C-reactive protein (CRP) in human serum and plasma was measured continuously after a sample collection in lithium heparin SARSTEDT Monovette ${ }^{\circledR}$ $4.7 \mathrm{ml}$ (orange top) using a standard immuno-turbidometric assay on the COBAS ${ }^{\circledR}$ INTEGRA system (the normal value is $<6 \mathrm{mg} / \mathrm{L}$ ). Blood leukocyte count (normal range $4.000-10.000 / \mu \mathrm{L}$ ) generally was carried out as a routine part of small or large blood counts after collection in EDTA Monovette ${ }^{\circledR} 2.7$ $\mathrm{mL}$ with flow cytometry.

Cardiovascular risk factors and acute and chronic comorbidities were analyzed in the four study groups. Comorbidity was considered the presence of one or more additional disorders existing simultaneously with a primary disease. The additional disorder may also be a behavioral or mental disorder. The inhalation therapy with salbutamol and atrovent was compared with systemic corticosteroid treatment among the four study groups.

Length of hospital stay was compared among the four study groups. The cumulative days were represented in the hospital stay of each individual patient over the 10-year period. The number of deaths during hospitalization was determined in each of the four study groups. Survival analyses were calculated by the day of discharge from the hospital after the number of deaths occurred based on the total number of patients in each group using the Kaplan-Meier method.

\section{Ethics statement}

All patients' data were anonymized before analysis. Saarland's Institutional Review Board approved the study. Due to the retrospective nature of the study protocol, the Medical Association of Saarland's Institutional Review Board waived the need for informed consent. Written informed patient consent was waived because of the retrospective analysis of the patients' medical records. 


\section{Statistical analysis}

Wherever appropriate, data are expressed via proportion, mean, and standard deviation. Ninety-five percent confidence intervals (CIs) were calculated for sex differences and patient deaths in each of the four groups. For medication with aspirin and statins, odds ratios were calculated for the likelihood that aspirin and statins prevented acute exacerbation of COPD or infection in patients with COPD. A chi-square test for four independent standard normal variables of two probabilities was used to compare sex difference, stages of COPD according to GOLD classification, infections, and indications for medication with aspirin and statins, medical treatment of patients with COPD, and deaths. One-way analysis of variance (ANOVA) for independent samples was performed to compare age differences, lung-function differences, and duration of hospital stays among all test groups, cardiovascular risk factors, and acute and chronic comorbidities. Survival rates for the four groups were calculated using the Kaplan-Meier method. All tests were expressed as two-tailed, and a $p$ value of $<0.05$ was considered statistically significant.

\section{Results}

This study followed 300 patients with COPD who had been treated at the Department of Internal Medicine, University Hospital of Saarland, Germany, from 2004 to 2014. The database included 514 medical reports for 300 patients with COPD. Those who met the inclusion criteria for the study had a mean age of $69 \pm 10$ years (206 men, 68.7\%, 95\% CI, 63.4-73.9; 94 women, 31.3\%, 95\% CI, 26.1-36.6). Eighteen cases of patients' medical data were excluded because some patients occupied more than one group at some time during the study, depending on newly identified indications or the discontinuation of aspirin or statins over the 10-year study period. In all, $64(21.3 \%, 95 \%$ CI, 0.17-0.26) patients with COPD were treated with aspirin, $12(4 \%, 95 \% \mathrm{CI}, 0.02-0.06)$ patients were treated with aspirin and statins, 51 (17\%, 95\% CI, 0.1-0.2) patients were treated with statins, and 173 $(57.7 \%, 95 \%$ CI, 0.5-0.6) patients were treated with neither aspirin nor statins (Table 1).

There were gender differences in the patients with COPD who were either taking or not taking aspirin or statins (Table 1). The male sex was more common in all study groups. The age of the patient was statistically significant, for those taking and those not taking aspirin or statins $(p=0.026$; Table 1$)$. Study results indicated no link between anti-infection effects and aspirin and statin use (Table 2). In contrast, the study found about a three-fold risk of infection among patients with COPD who took statins, which is statis- tically relevant $(p=0.0001$; Table 2$)$. The risk of infection for patients with COPD who took aspirin was not increased ( $p=0.382$; Table 2$)$. In addition, combining aspirin and statins did not have a positive effect on preventing acute exacerbations or infections $(\mathrm{p}=$ 0.136; Table 2). Conversely, the study detected an elevated number of acute exacerbations, acute urinary tract infections, and total number of infections in those who took and those who did not take aspirin and statins ( $\mathrm{p}<0.01$; Table 3$)$. Aspirin and statins were prescribed mainly for coronary artery disease (Table 4). There were marked differences in the GOLD stages for COPD and lung function among those in the study groups who were taking and those who were not taking aspirin and statins (Table 5). A comparison of inflammation values revealed no significant differences among the four study groups (Table 6). The duration of hospitalization did not differ among the study groups taking or not taking aspirin and statins $(\mathrm{p}=0.478$; Table 1$)$.

Although hypertension occurred in most study patients, a statistical significant difference was not observed among the cardiovascular risk factors in the four study groups (Table 7).

A marginal statistical difference was found between kidney diseases mainly among the aspirin, statins, and aspirin and statins groups (Table 8).

Table 1: Demographics and duration of hospital stay of patients with COPD with and without aspirin and statin use. The $p$ value was calculated by using the chi-square test $\left(\mathrm{X}^{2}\right)$ and one-way analysis of variance (ANOVA, $\eta^{2}$ ).

\begin{tabular}{llllll}
\hline & $\begin{array}{l}\text { Aspirin } \\
(\%)\end{array}$ & Statins (\%) & $\begin{array}{l}\text { Aspirin + } \\
\text { Statins (\%) }\end{array}$ & $\begin{array}{l}\text { Neither Aspirin p value } \\
\text { nor Statins (\%) }\end{array}$ & \\
\hline $\begin{array}{l}\text { Number of pa- } \\
\text { tients }\end{array}$ & 64 & 12 & 51 & 173 & \\
Male & $49(76.6)$ & $7(58.3)$ & $41(80.4)$ & $109(63.0)$ & $\mathbf{0 . 0 4 0} \times 2$ \\
$\begin{array}{l}\text { Female } \\
\text { Mean age }\end{array}$ & $15(23.4)$ & $5(41.7)$ & $10(23.08)$ & $64(37.0)$ & $\mathbf{0 . 0 4 0} \times 2$ \\
$\begin{array}{l}\text { Duration of hos- } \\
\text { pital stay } \\
\text { (mean of cumula- } \\
\text { tive days) }\end{array}$ & $10.6 \pm 8.9$ & $12.1 \pm 9.8$ & $14.4 \pm 28.4$ & $13.5 \pm 17.7$ & $0.478 \eta 2$ \\
\hline $\begin{array}{l}\text { Abbreviations: COPD: chronic obstructive pulmonary disease. Significant p values shown } \\
\text { in bold. }\end{array}$ & & & & & \\
\end{tabular}

Table 2: The total number of 514 cases with or without infection in 300 patients with COPD who took aspirin, statins, both, or neither during the study period.

\begin{tabular}{llllll}
\hline Medication & $\begin{array}{l}\text { Cases with } \\
\text { Infection } \\
(\mathrm{n}=315)(\%)\end{array}$ & $\begin{array}{l}\text { Cases with- } \\
\text { out Infection } \\
(\mathrm{n}=199)(\%)\end{array}$ & $\begin{array}{l}\text { Odds } \\
\text { Ratio }\end{array}$ & $\begin{array}{l}\text { 95\% Con- } \\
\text { fidence } \\
\text { Interval }\end{array}$ & p value \\
\hline Aspirin & $60(19.0)$ & $38(19.1)$ & 1.2 & $0.8-2$ & 0.382 \\
Statins & $42(13.3)$ & $10(5.0)$ & 3.3 & $1.6-6.8$ & $\mathbf{0 . 0 0 0 1}$ \\
Aspirin + statins & $66(21.0)$ & $36(18.1)$ & 1.4 & $0.9-2.3$ & 0.136 \\
$\begin{array}{l}\text { Neither aspirin nor } \\
\text { statins }\end{array}$ & $147(46.7)$ & $115(57.8)$ & 0.6 & $0.4-0.9$ & $\mathbf{0 . 0 1 4}$ \\
\hline
\end{tabular}

Abbreviations: COPD: chronic obstructive pulmonary disease. Significant $\mathrm{p}$ values shown in bold. 
Table 3: Comparison of all patients with COPD with various infections among the four study groups. The $p$ value was calculated by using the chi-square test $\left(x^{2}\right)$.

\begin{tabular}{|c|c|c|c|c|c|}
\hline \multirow[b]{2}{*}{ Infection } & \multicolumn{5}{|c|}{ Cases of COPD } \\
\hline & $\begin{array}{l}\text { Aspirin } \\
(\mathrm{n}=98) \\
(\%)\end{array}$ & $\begin{array}{l}\text { Statins } \\
(\mathrm{n}=52) \\
(\%)\end{array}$ & $\begin{array}{l}\text { Aspirin + } \\
\text { Statins (n } \\
=102)(\%)\end{array}$ & $\begin{array}{l}\text { Neither Aspi- } \\
\text { rin nor Statins } \\
(\mathrm{n}=262)(\%)\end{array}$ & $\begin{array}{l}\text { p value } \\
\left(x^{2}\right)\end{array}$ \\
\hline $\begin{array}{l}\text { Acute exacerbation of } \\
\text { COPD }\end{array}$ & $41(41.8)$ & $35(67.3)$ & $51(50.0)$ & $113(43.1)$ & 0.0009 \\
\hline Respiratory infections & $2(2.0)$ & $1(1.9)$ & $2(2.0)$ & $6(2.3)$ & 0.996 \\
\hline Pneumonia & $12(12.2)$ & $2(3.8)$ & $6(5.9)$ & $18(6.9)$ & 0.189 \\
\hline $\begin{array}{l}\text { Acute urinary tract } \\
\text { infection }\end{array}$ & $1(1.0)$ & $2(3.8)$ & $3(2.9)$ & 0 & 0.028 \\
\hline Erysipelas & 0 & $1(1.9)$ & 1 & 0 & 0.140 \\
\hline Sepsis & $1(1.0)$ & 0 & 0 & $2(0.8)$ & 0.713 \\
\hline $\begin{array}{l}\text { Other unspecified } \\
\text { infections }\end{array}$ & 0 & $1(1.9)$ & $3(2.9)$ & $8(3.1)$ & 0.371 \\
\hline Total number of cases & $57(58.2)$ & $42(80.8)$ & $65(63.7)$ & $147(56.1)$ & 0.008 \\
\hline
\end{tabular}

Table 4: Comparison of indications for aspirin and statins in various diseases. The $P$ value was calculated by using the chi-square test $\left(x^{2}\right)$.

\begin{tabular}{|c|c|c|c|c|c|}
\hline $\begin{array}{l}\text { Indication of aspirin } \\
\text { and statins }\end{array}$ & $\begin{array}{l}\text { Aspirin } \\
(\mathrm{n}=64) \\
(\%)\end{array}$ & $\begin{array}{l}\text { Statins } \\
(\mathrm{n}=12) \\
(\%)\end{array}$ & $\begin{array}{l}\text { Aspirin + } \\
\text { Statins } \\
(\mathrm{n}=51)(\%)\end{array}$ & $\begin{array}{l}\text { Neither } \\
\text { Aspirin } \\
\text { nor Statins } \\
(\mathrm{n}=173) \\
(\%)\end{array}$ & $\begin{array}{l}\mathrm{p} \text { value } \\
\left(x^{2}\right)\end{array}$ \\
\hline Coronary artery disease & $43(67.2)$ & $6(50.0)$ & $45(88.2)$ & 0 & $<0.0001$ \\
\hline Prior stroke & $6(9.4)$ & 0 & $4(7.8)$ & 0 & 0.0008 \\
\hline $\begin{array}{l}\text { Peripheral arterial } \\
\text { occlusive disease }\end{array}$ & $4(6.3)$ & 0 & $2(3.9)$ & 0 & 0.014 \\
\hline Atrial fibrillation & $4(6.3)$ & 0 & $2(3.9)$ & 0 & 0.014 \\
\hline Deep vein thrombosis & 0 & 0 & $3(5.9)$ & 0 & 0.002 \\
\hline Hypercholesterolemia & 0 & $1(8.3)$ & $2(3.9)$ & 0 & 0.004 \\
\hline Hyperlipidemia & 0 & $5(41.7)$ & $1(2.0)$ & 0 & $<0.0001$ \\
\hline
\end{tabular}

Significant $\mathrm{p}$ values shown in bold.

Table 5: Comparison of lung function in patients with COPD with and without aspirin and statin use. The $P$ value was calculated by using the chi-square test $\left(\mathrm{X}^{2}\right)^{*}$ and one-way analysis of variance $\left(\text { ANOVA, } \eta^{2}\right)^{\circ}$.

\begin{tabular}{llllll}
\hline Lung function & $\begin{array}{l}\text { Aspirin }(\mathrm{n}= \\
64)(\%)\end{array}$ & $\begin{array}{l}\text { Statins }(\mathrm{n} \\
=12)(\%)\end{array}$ & $\begin{array}{l}\text { Aspirin }+ \\
\text { Statins }(\mathrm{n}= \\
51)(\%)\end{array}$ & $\begin{array}{l}\text { Neither } \\
\text { Aspirin nor } \\
\text { Statins }(\mathrm{n}= \\
173)(\%)\end{array}$ & $\begin{array}{l}\mathrm{p} \text { value } \\
\left(x^{2}\right)^{*}\left(\mathrm{n}^{2}\right)^{\circ}\end{array}$ \\
\hline COPD GOLD 1 & $6(9.4)$ & $1(8.3)$ & $5(9.8)$ & $8(4.6)$ & $0.429^{*}$ \\
COPD GOLD 2 & $22(34.4)$ & $7(58.3)$ & $23(45.1)$ & $55(31.8)$ & $0.120^{*}$ \\
COPD GOLD 3 & $19(29.7)$ & $3(25.0)$ & $20(39.2)$ & $78(45.1)$ & $0.120^{*}$ \\
COPD GOLD 4 & $17(26.6)$ & $1(8.3)$ & $3(5.9)$ & $32(18.5)$ & $\mathbf{0 . 0 2 7}^{*}$ \\
FEV1\%VC & $70.7 \pm 17.3$ & $76.8 \pm 16.5$ & $76.7 \pm 20.2$ & $69.6 \pm 18.1$ & $0.078^{\circ}$ \\
FEV1 & $45 \pm 23.9$ & $59.3 \pm 15.8$ & $56.1 \pm 22.3$ & $48.4 \pm 29.5$ & $0.084^{\circ}$ \\
VC & $62.2 \pm 20.9$ & $68.2 \pm 13.2$ & $68.6 \pm 17$ & $64.1 \pm 20.7$ & $0.299^{\circ}$
\end{tabular}

Abbreviations: COPD: chronic obstructive pulmonary disease; GOLD: Global Initiative for Chronic Obstructive Lung Disease; FEV1\%VC: Tiffeneau index; FEV1: forced expiratory volume in the first second, VC: vital capacity. Significant p values shown in bold.

Table 6: Comparison of inflammation values in patients with COPD with and without aspirin and statin use. The $P$ value was calculated by using one-way analysis of variance (ANOVA, $\eta^{2}$ ).

\begin{tabular}{llllll}
\hline \multicolumn{6}{c}{ Cases of COPD } \\
\hline $\begin{array}{l}\text { Inflammation } \\
\text { values }\end{array}$ & $\begin{array}{l}\text { Aspirin } \\
(\mathrm{n}=98)\end{array}$ & $\begin{array}{l}\text { Statins } \\
(\mathrm{n}=52)\end{array}$ & $\begin{array}{l}\text { Aspirin }+ \\
\text { Statins }(\mathrm{n}= \\
102)\end{array}$ & $\begin{array}{l}\text { Neither Aspirin nor } \\
\text { Statins }(\mathrm{n}=262)\end{array}$ & $\begin{array}{l}\mathrm{p} \\
\text { value } \\
(\mathrm{n} 2)\end{array}$ \\
$\begin{array}{l}\text { C-reactive protein } \\
(\mathrm{CRP})(<6 \mathrm{mg} / \mathrm{L})\end{array}$ & $62.6 \pm 83$ & $54.3 \pm 74$ & $52.2 \pm 79.5$ & $47 \pm 72.6$ & 0.388 \\
$\begin{array}{l}\text { Leukocyte count } \\
(4.000-10.000 / \mu \mathrm{L})\end{array}$ & 10629.9 & 10615.4 & $9339.2 \pm$ & $10377 \pm 4589.4$ & 0.109 \\
\hline
\end{tabular}

Table 7: Cardiovascular risk factors in the four groups. The $p$ value was calculated by using one-way analysis of variance (ANOVA, $\eta^{2}$ ).

\begin{tabular}{|c|c|c|c|c|c|}
\hline & $\begin{array}{l}\text { Aspirin }(\mathrm{n} \\
=64)(\%)\end{array}$ & $\begin{array}{l}\text { Statins }(\mathrm{n} \\
=12)(\%)\end{array}$ & $\begin{array}{l}\text { Aspirin + } \\
\text { Statins (n } \\
=51)(\%)\end{array}$ & $\begin{array}{l}\text { Neither Aspi- } \\
\text { rin nor Statins } \\
(\mathrm{n}=173)(\%)\end{array}$ & $\begin{array}{l}p \\
\text { value } \\
\left(\eta^{2}\right)\end{array}$ \\
\hline $\begin{array}{l}\text { Cardiovascular risk } \\
\text { factors }\end{array}$ & & & & & 0.279 \\
\hline Hypertension & $55(85.9)$ & $10(83.3)$ & $46(90.2)$ & $147(85.0)$ & \\
\hline Diabetes & $13(20.3)$ & $3(25.0)$ & $18(35.3)$ & $49(28.3)$ & \\
\hline $\begin{array}{l}\text { Hypercholesterole- } \\
\text { mia }\end{array}$ & 0 & $1(8.3)$ & $2(3.9)$ & 0 & \\
\hline Hyperlipidemia & 0 & $5(41.7)$ & $1(2.0)$ & 0 & \\
\hline Obesity & $2(3.1)$ & $1(8.3)$ & $7(13.7)$ & $14(8.1)$ & \\
\hline Smoker & 0 & 0 & $1(2.0)$ & $5(2.9)$ & \\
\hline Former smoker & $6(9.4)$ & $1(8.3)$ & $6(11.8)$ & $18(10.4)$ & \\
\hline
\end{tabular}

Table 8: Comparison of acute comorbidities among the four study groups. The $p$ value was calculated by using one-way analysis of variance (ANOVA, $\eta^{2}$ ).

\begin{tabular}{|c|c|c|c|c|c|}
\hline \multicolumn{6}{|l|}{ Acute comorbidities } \\
\hline & $\begin{array}{l}\text { Aspirin }(n= \\
64)(\%)\end{array}$ & $\begin{array}{l}\text { Statins }(\mathrm{n} \\
=12)(\%)\end{array}$ & $\begin{array}{l}\text { Aspirin + } \\
\text { Statins }(\mathrm{n}= \\
51)(\%)\end{array}$ & $\begin{array}{l}\text { Neither } \\
\text { Aspirin nor } \\
\text { Statins (n= } \\
173)(\%)\end{array}$ & $\begin{array}{l}\mathrm{p} \\
\text { value } \\
\left(\mathrm{n}^{2}\right)\end{array}$ \\
\hline \multicolumn{5}{|c|}{ Cardiovascular disease } & 0.457 \\
\hline Acute heart failure & $55(85.9)$ & $12(100)$ & $38(74.5)$ & $148(85.5)$ & \\
\hline Anemia & $2(3.1)$ & 0 & $3(5.9)$ & $8(4.6)$ & \\
\hline Cardiac arrhythmia & $4(6.3)$ & 0 & $2(3.9)$ & 0 & \\
\hline $\begin{array}{l}\text { Cardiac decom- } \\
\text { pensation }\end{array}$ & $7(10.9)$ & $2(16.7)$ & $6(11.8)$ & $25(14.5)$ & \\
\hline $\begin{array}{l}\text { Circulatory col- } \\
\text { lapse }\end{array}$ & $2(3.1)$ & 0 & 0 & $1(0.6)$ & \\
\hline $\begin{array}{l}\text { Coronary artery } \\
\text { disease }\end{array}$ & $43(67.2)$ & $6(50.0)$ & $45(88.2)$ & 0 & \\
\hline $\begin{array}{l}\text { Derailed blood } \\
\text { pressure }\end{array}$ & $6(9.4)$ & 0 & $7(13.7)$ & $17(9.8)$ & \\
\hline Shock & $1(1.6)$ & $1(8.3)$ & $1(2.0)$ & $4(2.3)$ & \\
\hline Syncope & $2(3.1)$ & $1(8.3)$ & $1(2.0)$ & $7(4.0)$ & \\
\hline \multicolumn{5}{|c|}{ Pulmonary disease } & 0.459 \\
\hline $\begin{array}{l}\text { Acute respiratory } \\
\text { failure }\end{array}$ & 0 & 0 & $2(3.9)$ & $5(2.9)$ & \\
\hline Pulmonary edema & $2(3.1)$ & 0 & 0 & $1(0.6)$ & \\
\hline \multicolumn{5}{|c|}{ Gastrointestinal diseases } & 0.089 \\
\hline Duodenal ulcer & 0 & 0 & 0 & $1(0.6)$ & \\
\hline $\begin{array}{l}\text { Gastrointestinal } \\
\text { bleeding }\end{array}$ & $2(3.1)$ & 0 & 0 & $2(1.2)$ & \\
\hline Refluxesophagitis & 0 & 0 & $1(2.0)$ & $2(1.2)$ & \\
\hline Kidney disease & & & & & 0.049 \\
\hline $\begin{array}{l}\text { Acute kidney } \\
\text { injury }\end{array}$ & $3(4.7)$ & 0 & $1(2.0)$ & $5(2.9)$ & \\
\hline $\begin{array}{l}\text { Acute urinary tract } \\
\text { infection }\end{array}$ & $4(6.3)$ & $1(8.3)$ & $1(2.0)$ & $12(6.9)$ & \\
\hline Macrohematuria & 1 (1.6) & $1(8.3)$ & 0 & $3(1.7)$ & \\
\hline $\begin{array}{l}\text { Water-electrolyte } \\
\text { imbalance }\end{array}$ & 0 & 0 & $1(2.0)$ & $2(1.2)$ & \\
\hline Thyroid disease & & & & & 0.252 \\
\hline Hyperthyroidism & $1(1.6)$ & 0 & $1(2.0)$ & $3(1.7)$ & \\
\hline Hypothyroidism & $3(4.7)$ & 0 & $4(7.8)$ & $9(5.2)$ & \\
\hline Diverse disease & & & & & 0.002 \\
\hline Gout attack & 0 & 0 & 0 & $1(0.6)$ & \\
\hline Delirium & 0 & 0 & $1(2.0)$ & $3(1.7)$ & \\
\hline Fall & $1(1.6)$ & 0 & $1(2.0)$ & $3(1.7)$ & \\
\hline Leg ulcers & 0 & 0 & 0 & $1(0.6)$ & \\
\hline Nosebleed & 0 & 0 & $1(2.0)$ & $1(0.6)$ & \\
\hline
\end{tabular}

After chronic diseases were compared, statistically significant differences were detected between gastrointestinal, genitourinary, orthopedic, and psychiatric disorders between the groups (Table 9). 
Table 9: Comparison of chronic comorbidities among the four study groups. The $p$ value was calculated by using one-way analysis of variance (ANOVA, $\eta^{2}$ ).

\begin{tabular}{|c|c|c|c|c|c|}
\hline \multicolumn{6}{|l|}{ Chronic comorbidities } \\
\hline & $\begin{array}{l}\text { Aspirin }(\mathrm{n} \\
=64)(\%)\end{array}$ & $\begin{array}{l}\text { Statins }(\mathrm{n} \\
=12)(\%)\end{array}$ & $\begin{array}{l}\text { Aspirin + } \\
\text { Statins (n } \\
=51)(\%)\end{array}$ & $\begin{array}{l}\text { Neither } \\
\text { Aspirin } \\
\text { nor Statins } \\
(\mathrm{n}= \\
173)(\%)\end{array}$ & $\begin{array}{l}p \text { value } \\
\left(\mathrm{n}^{2}\right)\end{array}$ \\
\hline \multicolumn{5}{|l|}{ Cardiovascular disease } & 0.178 \\
\hline Aneurysm & $1(1.6)$ & 0 & $2(3.9)$ & $3(1.7)$ & \\
\hline Cardiac valvular defect & $28(43.8)$ & $8(66.7)$ & $27(52.9)$ & $85(49.1)$ & \\
\hline Cardiomyopathy & $1(1.6)$ & 0 & $1(2.0)$ & $4(2.3)$ & \\
\hline Carotid stenosis & 0 & 0 & $1(2.0)$ & $4(2.3)$ & \\
\hline $\begin{array}{l}\text { Chronic venous insuffi- } \\
\text { ciency }\end{array}$ & $1(1.6)$ & 0 & $2(3.9)$ & $6(3.5)$ & \\
\hline Cor pulmonale & $5(7.8)$ & 0 & $4(7.8)$ & $15(8.7)$ & \\
\hline $\begin{array}{l}\text { Hypertensive heart } \\
\text { disease }\end{array}$ & $4(6.3)$ & $1(8.3)$ & $1(2.0)$ & $8(4.6)$ & \\
\hline Pacemaker & $7(10.9)$ & 0 & $9(17.6)$ & 20 (11.6) & \\
\hline $\begin{array}{l}\text { Peripheral arterial } \\
\text { occlusive disease }\end{array}$ & $4(6.3)$ & 0 & $2(3.9)$ & 0 & \\
\hline State after syncope & $2(3.1)$ & 0 & 0 & $2(1.2)$ & \\
\hline \multicolumn{5}{|l|}{ Pulmonary disease } & 0.073 \\
\hline Asthma & 0 & 0 & $1(2.0)$ & $1(0.6)$ & \\
\hline Emphysema & $2(3.1)$ & 0 & 0 & $1(0.6)$ & \\
\hline $\begin{array}{l}\text { Obstructive sleep apnea } \\
\text { syndrome }\end{array}$ & $1(1.6)$ & 0 & $1(2.0)$ & $2(1.2)$ & \\
\hline State after tuberculosis & $1(1.6)$ & $1(8.3)$ & $1(2.0)$ & $3(1.7)$ & \\
\hline \multicolumn{5}{|c|}{ Gastrointestinal disease } & 0.009 \\
\hline Appendectomy & $4(6.3)$ & 0 & $7(13.7)$ & $11(6.4)$ & \\
\hline Cholecystectomy & $9(14.1)$ & $3(25.0)$ & $3(5.9)$ & $20(11.6)$ & \\
\hline Colon carcinoma & 0 & 0 & $1(2.0)$ & $2(1.2)$ & \\
\hline Colonic diverticula & $2(3.1)$ & $1(8.3)$ & $2(3.9)$ & $6(3.5)$ & \\
\hline Fatty liver & 0 & 0 & 0 & $1(0.6)$ & \\
\hline Gallbladder stones & 0 & 0 & 0 & $2(1.2)$ & \\
\hline Liver cysts & 0 & 0 & 0 & $1(0.6)$ & \\
\hline Pancreatic disease & 0 & 0 & 0 & $1(0.6)$ & \\
\hline Splenectomy & 0 & 0 & 0 & $1(0.6)$ & \\
\hline $\begin{array}{l}\text { State after bowel sur- } \\
\text { gery }\end{array}$ & $1(1.6)$ & 0 & $3(5.9)$ & $8(4.6)$ & \\
\hline State after hepatitis B & $3(4.7)$ & $1(8.3)$ & $1(2.0)$ & $3(1.7)$ & \\
\hline $\begin{array}{l}\text { State after hernia opera- } \\
\text { tion }\end{array}$ & 0 & 0 & $2(3.9)$ & $4(2.3)$ & \\
\hline Stomach cancer & $1(1.6)$ & $1(8.3)$ & 0 & $2(1.2)$ & \\
\hline \multicolumn{5}{|l|}{ Kidney disease } & 0.288 \\
\hline Chronic renal failure & $15(23.4)$ & $1(8.3)$ & $11(21.6)$ & $40(23.1)$ & \\
\hline Contracted kidney & 0 & 0 & 0 & $1(0.6)$ & \\
\hline Diabetic nephropathy & $2(3.1)$ & $1(8.3)$ & $1(2.0)$ & $4(2.3)$ & \\
\hline Nephrectomy & $2(3.1)$ & $1(8.3)$ & 0 & $3(1.7)$ & \\
\hline Renal adenoma & $1(1.6)$ & $1(8.3)$ & 0 & $1(0.6)$ & \\
\hline Renal artery stenosis & $1(1.6)$ & 0 & $1(2.0)$ & $2(1.2)$ & \\
\hline Renal cysts & $1(1.6)$ & $1(8.3)$ & $1(2.0)$ & $7(4.0)$ & \\
\hline State after kidney stones & $1(1.6)$ & $1(8.3)$ & 0 & $2(1.2)$ & \\
\hline \multicolumn{5}{|c|}{ Genitourinary system disease } & 0.039 \\
\hline $\begin{array}{l}\text { Benign prostate hyper- } \\
\text { plasia }\end{array}$ & $5(7.8)$ & 0 & $1(2.0)$ & $8(4.6)$ & \\
\hline Cystectomy & 0 & 0 & $1(2.0)$ & $1(0.6)$ & \\
\hline Prostate cancer & $1(1.6)$ & 0 & 0 & $3(1.7)$ & \\
\hline Prostatectomy & $3(4.7)$ & 0 & $4(7.8)$ & $8(4.6)$ & \\
\hline $\begin{array}{l}\text { State after bladder } \\
\text { carcinoma }\end{array}$ & $1(1.6)$ & $1(8.3)$ & 0 & $2(1.2)$ & \\
\hline \multicolumn{5}{|l|}{ Thyroid disease } & 1.0 \\
\hline Struma & 0 & 0 & $2(3.9)$ & $3(1.7)$ & \\
\hline Strumectomy & 0 & 0 & $2(3.9)$ & $3(1.7)$ & \\
\hline \multicolumn{5}{|l|}{ Nervous system disorders } & 0.151 \\
\hline Chronic lumbago & 0 & 0 & 0 & $1(0.6)$ & \\
\hline Disc herniation & $3(4.7)$ & $1(8.3)$ & $2(3.9)$ & $4(2.3)$ & \\
\hline Parkinson disease & $2(3.1)$ & $1(8.3)$ & $1(2.0)$ & $6(3.5)$ & \\
\hline Polyneuropathy & 0 & 0 & $2(3.9)$ & $4(2.3)$ & \\
\hline Spinal canal stenosis & 0 & 0 & $1(2.0)$ & $2(1.2)$ & \\
\hline State after stroke & $6(9.4)$ & 0 & $4(7.8)$ & 0 & \\
\hline
\end{tabular}

Chronic comorbidities

Aspirin (n Statins ( $\mathrm{n}$ Aspirin + Neither $p$ value
$=64)(\%) \quad=12)(\%) \quad$ Statins $\left(\mathrm{n}\right.$ Aspirin $\quad\left(\mathrm{n}^{2}\right)$ $=51)(\%) \quad$ nor Statins ( $(\mathrm{n}=$

\begin{tabular}{llllll} 
& & & \multicolumn{3}{c}{$(\mathrm{n}=$} \\
& & & & \\
\hline $\begin{array}{l}\text { Orthopedic disorders } \\
\text { Osteoarthritis }\end{array}$ & $2(3.1)$ & $1(8.3)$ & $2(3.9)$ & $12(6.9)$ &
\end{tabular}

$\begin{array}{lllll}\text { Rheumatism } & 1(1.6) & 0 & 1(2.0) & 3(1.7)\end{array}$

Spondyloarthropathy $\begin{array}{lllll}0 & 0 & 1(2.0) & 2(1.2)\end{array}$

State after bone fracture $3(4.7) \quad 2(16.7) \quad 4(7.8) \quad 13(7.5)$

Psychiatric disorders

$\begin{array}{lllll}\text { Dementia } & 3(4.7) & 1(8.3) & 0 & 2(1.2)\end{array}$

$\begin{array}{lllll}\text { Depression } & 3(4.7) & 0 & 1(2.0) & 3(1.7)\end{array}$

Ear, nose, and throat disease

$\begin{array}{lllll}\text { Nasal polypectomy } & 2(3.1) & 0 & 0 & 1(0.6)\end{array}$

$\begin{array}{lllll}\text { Tonsillectomy } & 3(4.7) & 0 & 4(7.8) & 6(3.5)\end{array}$

Skin disorders $\quad 0.577$

$\begin{array}{lllll}\text { Allergy } & 2(3.1) & 0 & 5(9.8) & 7(4.0)\end{array}$

$\begin{array}{lllll}\text { State after post-herpes } & 0 & 0 & 0 & 1(0.6)\end{array}$

zoster

Ophthalmologic diseases

$\begin{array}{lllll}\text { Cataract } & 3(4.7) & 0 & 5(9.8) & 8(4.6)\end{array}$

Various ocular diseases $2(3.1) \quad 0 \quad 0 \quad 6(3.5)$

Gynecological disorders

$\begin{array}{lllll}\text { State after breast cancer } & 1(1.6) & 0 & 0 & 2(1.2)\end{array}$

$\begin{array}{lllll}\text { Hysterectomy } & 3(4.7) & 2(16.7) & 2(3.9) & 7(4.0)\end{array}$

0.029

0.543

0.089

0.422

Significant $\mathrm{p}$ values shown in bold

Table 10: Medical treatment of patients with COPD in cases of exacerbation and pneumonia. The $p$ value was calculated by using the chi-square test $\left(x^{2}\right)$.

Medical treatment of patients with COPD

Number of cases

\begin{tabular}{|c|c|c|c|c|c|}
\hline & $\begin{array}{l}\text { Aspirin } \\
(\mathrm{n}=98) \\
(\%)\end{array}$ & $\begin{array}{l}\text { Statins } \\
(\mathrm{n}=52) \\
(\%)\end{array}$ & $\begin{array}{l}\text { Aspirin }+ \\
\text { Statins } \\
(\mathrm{n}=102) \\
(\%)\end{array}$ & $\begin{array}{l}\text { Neither Aspirin } \\
\text { nor Statins } \\
(\mathrm{n}=262)(\%)\end{array}$ & $\begin{array}{l}\mathrm{p} \\
\text { value } \\
(\times 2)\end{array}$ \\
\hline $\begin{array}{l}\text { Inhalation of salbutamol } \\
\text { and ipratropium bromide }\end{array}$ & $55(56.1)$ & $38(73.1)$ & $59(57.8)$ & $137(52.3)$ & 0.051 \\
\hline Systemic steroids & $23(23.5)$ & $2(3.8)$ & 14 (13.7) & 36 (13.7) & 0.011 \\
\hline
\end{tabular}

Most patients with COPD who experienced pneumonia and exacerbations received inhalation therapy with salbutamol and atrovent without statistically significant differences between the study groups (Table 10). Systemic steroid therapies were not performed in most patients with COPD with a statistically significant difference (Table 10).

Three $(4.7 \%, 95 \% \mathrm{CI},-0.005$ to 0.1$)$ patients with COPD who took aspirin died, no patients who took statins died, one $(2 \%, 95 \% \mathrm{CI},-0.02-0.06)$ patient who took aspirin and statins died, and eight $(4.6 \%, 95 \% \mathrm{CI}$, $0.01-0.08)$ patients who took neither aspirin nor statins died; none had statistical significance $(\mathrm{p}=$ 0.727). Since there was no statistical relevance, the reasons for the deaths were not examined in detail. The survival rate was $95.3 \%(95 \% \mathrm{CI}, 0.9-1)$ in patients with COPD who took aspirin, $100 \%$ (95\% CI, 1-1) in patients with COPD who took statins, $98 \%$ (95\% CI, 0.9-1) in patients with COPD who took aspirin and 
statins, and $95.4 \%(95 \% \mathrm{CI}, 0.9-1)$ in patients with COPD who took neither aspirin nor statins.

\section{Discussion}

In this study, the number of acute exacerbations and infections increased in patients with COPD who took aspirin and statins compared with those who took neither aspirin nor statins. According to these results, aspirin and statins do not prevent exacerbation and infection in patients with COPD.

Aspirin inhibits thromboxane-dependent platelet activation. ${ }^{10}$ Aspirin permanently inhibits cyclooxygenase (COX-1) on platelets, thus decreasing thromboxane A2, an effective vasoconstrictor anti-platelet activator. ${ }^{11}$ Aspirin has been used in primary and secondary prevention of coronary artery disease. In this study, aspirin and statins were prescribed mainly to patients with coronary artery disease. It is well-known that anti-platelet therapy with aspirin decreases the frequency of adverse cardiovascular events in patients with acute coronary syndromes. ${ }^{12-15}$

Pre-operative use of aspirin and clopidogrel was reported in a previous study that found increased risk of infection after coronary-artery bypass surgery. ${ }^{16}$ This discovery spurred further investigations to clarify the dangers and benefits of uninterrupted dual anti-platelet therapy in post-operative patients and the influence of platelet inhibition on infections in patients at increased risk of infection.

Aspirin can exacerbate respiratory disease. ${ }^{17-19}$ Regardless of aspirin use to prevent adverse cardiovascular events, possible resistance to the drug aspirin has been reported. ${ }^{20}$ However, this study did not find exacerbation of respiratory disease or resistance to aspirin.

Aspirin has anti-aggregating and anti-inflammatory effects. ${ }^{21}$ Summarized data showed that pneumonia may generate acute coronary syndrome as a consequence of inflammatory responses and pro-thrombotic changes in patients with pneumonia. Therefore, one study examined the use of aspirin to reduce the risk of acute coronary syndrome in patients with pneumonia. ${ }^{21}$ In addition; aspirin may be useful for the primary prevention of acute coronary syndrome in patients with pneumonia. ${ }^{21}$ A study has shown that aspirin was advantageous in decreasing acute coronary syndrome and cardiovascular mortality in patients with pneumonia. ${ }^{20}$

Another study examined the correlation between acute respiratory infection and acute coronary syndrome in patients with infection and acute coronary syndrome considered to be caused by platelet aggregation or aspirin failure. ${ }^{22}$ Patients with urinary tract infections and pneumonia had more aggregates than did patients without an infection. ${ }^{22}$ Aspirin failure was more common in patients with pneumonia than in those without an infection. The CRP values of the study patients were independently connected with platelet aggregation and aspirin failure. ${ }^{22}$ Infection during acute coronary syndrome led to more obvious platelet aggregation. Aspirin failure was found more often in patients with pneumonia. The study also found that CRP was a neutral predictor of platelet aggregation and aspirin failure in the context of an acute coronary syndrome. ${ }^{22}$ In the present study, CRP values and leucocyte counts increased unremarkably in the study population. However, platelet counts were not investigated further.

Sepsis is an acute inflammatory illness associated with notable morbidity and mortality. Due to the absence of special, established therapies for sepsis, avoidance is of great importance. Secondary to their pleiotropic effects, statins have been recognized for their role in preventing non-atherosclerotic disorders. In addition, platelets play an important part in the inflammatory cascade of sepsis. Data indicate that anti-platelet therapy with aspirin may reduce platelets' undesirable effects. ${ }^{23}$ In addition, previous studies have showed that patients who use statins have reduced incidence of sepsis. ${ }^{23}$ Since aspirin and statins have special effects on the immune system and inflammatory pathways, they may be sustainable medical alternatives for preventing sepsis and cardiovascular disease. ${ }^{23}$ However, the present study detected little sepsis in patients with COPD who either took or did not take aspirin or statins. Therefore, the use of aspirin and statins to prevent sepsis and septic shock cannot be confirmed.

One previous study postulated a decreased risk of COPD exacerbations with statins. ${ }^{24}$ Statins have various anti-inflammatory effects in addition to their lipid-lowering capacity. Another study examined the number of COPD exacerbations and intubations in patients who took statins. ${ }^{25}$ Patients with COPD who took statins had fewer exacerbations and intubations than patients with COPD who did not take statins..$^{25}$

Another study of patients with COPD examined the role of statins on the outcome of exacerbations and found that statin treatment was associated with a lowered risk of exacerbations. ${ }^{26}$

A randomized, controlled study of simvastatin versus a placebo examined simvastatin's role in preventing COPD exacerbations, ${ }^{27}$ and showed that the mean number of exacerbations per person-year was similar in the simvastatin and placebo groups. In addition, the median number of days before the first exacerbation was similar. In both groups, the number of nonfatal, serious, adverse events per person-year was similar. There were nearly similar numbers of 
deaths in the placebo and simvastatin groups. Simvastatin did not influence exacerbation rates or the time before the first exacerbation in patients with COPD who were at high risk for exacerbations. ${ }^{27}$ This study's results were similar to those of the present study, which found no connection between statin use and the reduced rate of exacerbations. Quite the contrary, the risk of exacerbation increased in patients with COPD who took statins. However, the mortality rate in patients with COPD who took aspirin and statins was lower compared to patients with COPD who took neither.

\section{Study limitations}

This study investigated only patients with COPD in the Department of Internal Medicine, not other parts of the hospital. The study did not include data on any treatment for exacerbations in study population members performed in other hospitals. Indications for the use of aspirin and statins changed in various cases during the study's 10-year observation period; therefore, some patients were not evaluated in more than one study group. The study did not investigate in detail reasons for discontinued use of aspirin or statins. Patients who were classified as nonusers of aspirin and statins could take the medications from primary care outside the hospital or from other medical departments in the hospital. Patients identified as those without COPD exacerbation or infection could encounter these events either in primary care outside the hospital or in other medical departments in the hospital. Important confounders were not taken into account, which might cause a spurious observed association. This study examined only the crude relationship between statin, aspirin use, and the risk of infection, without considering the imbalanced COPD severity and other important confounders such as comedications that might be relevant to infection.

\section{Conclusions}

This study was not able to confirm a decreased incidence of exacerbation in patients with COPD treated with aspirin or statins. Study data did not show that the anti-inflammatory effects of aspirin or statins reduced exacerbation or infection rates in patients with COPD.

\section{Competing Interests}

The author has declared that no competing interest exists.

\section{References}

1. Vestbo J. COPD: definition and phenotypes. Clin Chest Med. 2014;35:1-6.

2. Murărescu ED, Mitrofan EC, Mihailovici MS. Chronic obstructive pulmonary disease in a new concept. Rom J Morphol Embryol. 2007;48:207-14.

3. Romagnoli M, Fabbri LM. Chronic obstructive pulmonary disease: definition and classification of severity. Ann Ist Super Sanita. 2003;39:461-6.
4. Trigueros Carrero JA. How should we define and classify exacerbations in chronic obstructive pulmonary disease? Expert Rev Respir Med. 2013;7:33-41.

5. Kaczmarek P, Sladek K, Skucha W, Rzeszutko M, Iwaniec T, Dziedzina S, et al. The influence of simvastatin on selected inflammatory markers in patients with chronic obstructive pulmonary disease. Pol Arch Med Wewn. 2010;120:11-7.

6. Huang CC, Chan WL, Chen YC, Chen TJ, Chou KT, Lin SJ, et al. Statin use and hospitalization in patients with chronic obstructive pulmonary disease: a nationwide population-based cohort study in Taiwan. Clin Ther. 2011;33:1365-70

7. Harrison MT, Short P, Williamson PA, Singanayagam A, Chalmers JD, Schembri S. Thrombocytosis is associated with increased short and long term mortality after exacerbation of chronic obstructive pulmonary disease: a role for antiplatelet therapy? Thorax. 2014;69:609-15.

8. Winning J, Reichel J, Eisenhut Y, Hamacher J, Kohl M, Deigner HP, et al. Anti-platelet drugs and outcome in severe infection: clinical impact and underlying mechanisms. Platelets. 2009;20:50-7.

9. Vestbo J, Hurd SS, Agustí AG, Jones PW, Vogelmeier C, Anzueto A, et al. Global strategy for the diagnosis, management, and prevention of chronic obstructive pulmonary disease: GOLD executive summary. Am J Respir Crit Care Med. 2013; 187:347-65.

10. Patrono C, García Rodríguez LA, Landolfi R, Baigent C. Low-dose aspirin for the prevention of atherothrombosis. N Engl J Med. 2005;353:2373-83.

11. Patrono C, Coller B, FitzGerald GA, Hirsh J, Roth G. Platelet-active drugs: the relationships among dose, effectiveness, and side effects: the Seventh ACCP Conference on Antithrombotic and Thrombolytic Therapy. Chest. 2004;126:234S-264S.

12. Mahony C. Effect of aspirin on myocardial ischemia. Am J Cardiol. 1989;64:387-9.

13. Mehta JL. Emerging options in the management of myocardial ischemia. Am J Cardiol. 1994;73:18A-27A.

14. Cheng JW. Updates in antiplatelet agents used in cardiovascular diseases. J Cardiovasc Pharmacol Ther. 2013;18:514-24.

15. Ittaman SV, Vanwormer JJ, Rezkalla SH. The role of aspirin in the prevention of cardiovascular disease. Clin Med Res. 2014 Feb 26.

16. Blasco-Colmenares E, Perl TM, Guallar E, Baumgartner WA, Conte JV, Alejo $\mathrm{D}$, et al. Aspirin plus clopidogrel and risk of infection after coronary artery bypass surgery. Arch Intern Med. 2009;169:788-96.

17. White AA, Hope AP, Stevenson DD. Failure to maintain an aspirin-desensitized state in a patient with aspirin-exacerbated respiratory disease. Ann Allergy Asthma Immunol. 2006;97:446-8.

18. Macy E, Bernstein JA, Castells MC, Gawchik SM, Lee TH, Settipane RA, et al. Aspirin challenge and desensitization for aspirin-exacerbated respiratory disease: a practice paper. Ann Allergy Asthma Immunol. 2007;98:172-4.

19. Mitchell JE, Skypala I. Aspirin and salicylate in respiratory disease. Rhinology. 2013;51:195-205.

20. Grinstein J, Cannon CP. Aspirin resistance: current status and role of tailored therapy. Clin Cardiol. 2012;35:673-81.

21. Oz F, Gul S, Kaya MG, Yazici M, Bulut I, Elitok A, et al. Does aspirin use prevent acute coronary syndrome in patients with pneumonia: multicenter prospective randomized trial. Coron Artery Dis. 2013;24:231-7.

22. Modica A, Karlsson F, Mooe T. Platelet aggregation and aspirin non-responsiveness increase when acute coronary syndrome is complicated by an infection. J Thromb Haemost. 2007;5:507-11.

23. Sanchez MA, Thomas $\mathrm{CB}, \mathrm{O}^{\prime} \mathrm{Neal} \mathrm{HR}$. Do aspirin and statins prevent severe sepsis? Curr Opin Infect Dis. 2012;25:345-50.

24. Wang MT, Lo YW, Tsai CL, Chang LC, Malone DC, Chu CL, et al. Statin use and risk of COPD exacerbation requiring hospitalization. Am J Med. 2013:126:598-606.

25. Blamoun Al, Batty GN, DeBari VA, Rashid AO, Sheikh M, Khan MA. Statins may reduce episodes of exacerbation and the requirement for intubation in patients with COPD: evidence from a retrospective cohort study. Int J Clin Pract. 2008;62:1373-8.

26. Bartziokas K, Papaioannou AI, Minas M, Kostikas K, Banya W, Daniil ZD, et al. Statins and outcome after hospitalization for COPD exacerbation: a prospective study. Pulm Pharmacol Ther. 2011;24:625-31.

27. Criner GJ, Connett JE, Aaron SD, Albert RK, Bailey WC, Casaburi R, et al. Simvastatin for the prevention of exacerbations in moderate-to-severe COPD. N Engl J Med. 2014;370:2201-10. 